\title{
Electrocautery Versus Scalpel in Women Undergoing Primary Cesarean Section and Neonatal Outcomes
}

\author{
Esra ISCI BOSTANCI' ${ }^{1}$, Ismail GULER ${ }^{2}$, Funda CEVHER AKDULUM ${ }^{2}$, Mehmet Anil ONAN ${ }^{3}$ \\ Ankara, Turkey
}

\begin{abstract}
OBJECTIVE: The aim of this study is to evaluate the postoperative wound infection rate, newborn effect and, the effect of skin to peritoneum incision time between diathermy and scalpel for the operation time during primer cesarean section cases.

STUDY DESIGN: A retrospective study was carried out at Gazi University Hospital. A total of 74 patients met inclusion criteria in this study but 6 patients were excluded in electrocautery group for not to come controls, group I patients, $n=37$, underwent operation via scalpel incision and group II patients, $n=31$, underwent operation by diathermy incision. The main outcome measures were the operation time, postoperative wound infection rate, scar character, neonatal Apgar scores, and need of neonatal intensive care unit.
\end{abstract}

RESULTS: There were no significant differences between newborn Apgar scores ( $1^{\text {st }}$ and $5^{\text {th }}$ minutes), wound infections, and operation times ( $p=0.35, p=0.69, p=0.32$, respectively).

CONCLUSION: Related to findings, it could be suggested that diathermy might be an alternative to the scalpel in Pfannenstiel incisions contrary to the old belief about its high infection rates.

Keywords: Diathermy, Neonatal apgar scores, Primary cesarean section, Scalpel, Wound infection

Gynecol Obstet Reprod Med 2020;26(3):184-187

\section{Introduction}

Cesarean section (CS) is the most preferable procedure performed on women in the worldwide. Abdominal incision

${ }^{1}$ The Department of Gynecologic Oncology, Etlik Zubeyde Hanim Women's Health Training and Research Hospital, Ankara, Turkey

2 The Department of Obstetrics and Gynecology, Gazi University Medical Faculty Hospital, Ankara, Turkey

${ }^{3}$ The Department of Gynecologic Oncology, Gazi University Medical Faculty Hospital, Ankara, Turkey

Address of Correspondence: Esra Isci Bostanci

The Department of Gynecologic Oncology, Etlik Zubeyde Hanim Women's Health

Training and Research Hospital, Ankara, Turkey

dresrai@yahoo.com.tr

Submitted for Publication: $\quad$ 24.02.2019

Accepted for Publication: $\quad$ 19.07.2019

ORCID IDs of the authors: $\quad$ EI.B: 0000-0002-7703-7608,

IG: 0000-0002-8098-2483, FCA: 0000-0003-2285-7112, MAO: 0000-0001-7643-1585

\begin{tabular}{|c|c|}
\hline \begin{tabular}{c} 
Quick Response Code: \\
\cline { 2 - 2 } \\
\cline { 2 - 2 }
\end{tabular} & $\begin{array}{c}\text { Access this article online } \\
\text { Website: www.gorm.com.tr } \\
\text { e- mail: info@gorm.com.tr }\end{array}$ \\
\cline { 2 - 3 } & DOI:10.21613/GORM.2019.921 \\
\hline
\end{tabular}

How to cite this article: Isci Bostanci E. Guler I. Cevher Akdulum F. Onan MA. Electrocautery Versus Scalpel In Women Undergoing Primary Cesarean Section And Neonatal Outcomes. Gynecol Obstet Reprod Med 2020; 26(3): 184-187 types and instrumentations should show variety according to the situation. The techniques used to perform CS should depend on many factors including the clinical situation correlated with mother and fetus and also the preferences of the operator (1). Regardless of those factors, the safest and appropriate procedure must be used by the obstetrician.

The high-frequency electric surgical knife is the most common instrument in surgical operations since use by Harvey Cushing in 1926 (2). With the increasing use of electrosurgical tools for tissue dissection, cutting, and homeostasis, fear of excessive scarring and impair wound healing have decreased the widespread use of diathermy (3). The concern of increased infection rates by the use of electrocautery and scar outcome lead to compare with a scalpel that provides an incision with well-wound healing (4). Recently published studies have assessed electrocautery and scalpel incisions according to complications such as; blood loss, early postoperative pain, and post-operative wound infection rates (5). According to the literature, we found any single study that points the usage of electrocautery in primer cesarean section exclusively so as to figure out the post-operative wound infection rate, newborn effect, and scar character.

This study compared electrocautery and steel scalpel incision in terms of incision time, postoperative wound infection rate, and scar character. 


\section{Material and Method}

Women admitted for delivery at the obstetrical unit of the Gazi University Hospital in who had received prenatal care and who had no homeostatic problems, were included in the study. Inclusion criteria were dependent on the need to perform a CS, which was determined by the obstetrical team at any point during labor. Inclusion criteria included non-urgent primary CS in who had not allergic or immunodeficiency syndromes. Patients in whom a CS was indicated as an emergency procedure (e.g., for treatment of placental abruption), patients who have used antibiotics in the 30 days preceding delivery, patients who had undergone more than one previous $\mathrm{CS}$, and women who had high-risk factors for the fetus and themselves were excluded from the study. Signed informed consent was obtained from each participant at the time of operation and the study was conducted in accordance with the 1964 Helsinki Declaration. The study was performed with the approval of the Ethics Committee of the Faculty of Medicine, Gazi University (No: 101, 12.02.2018).

The first case was allocated a group on the basis of the draw and thereafter patients were divided into two groups alternately. A total of 74 patients were included in this study. 6 patients were excluded in the electrocautery group for not to come controls. Group I patients, $n=37$, underwent operation via scalpel incision. Group II patients, $n=31$, underwent operation via diathermy incision.

All of the women underwent $\mathrm{C} / \mathrm{S}$ under spinal anesthesia. Intravenous medication (cephalosporin) was given to whole patients prophylactically in the form of ceftriaxone intravenous 1 gram at the beginning of the induction of anesthesia. Antibiotic was given 12 hourly for a period of 3 days. Diclofenac $75 \mathrm{mg}$ intramuscular injection was given two times per day, pursued by diclofenac oral $50 \mathrm{mg}$ three times a day for 3 days. Subcutaneous tissue was sutured using vicryl (polyglactin 910 Suture) 2/0 and skin closure was done using non-absorbable monofilament suture $2 / 0$.

Assessment of wound infections was done by sending wound discharge for culture. Wound infection was graded according to the Southampton wound scoring system: Grade I; normal healing with mild bruising or erythema, Grade II; erythema plus other signs of inflammation, Grade III; clear or serosanguinous discharge, and Grade IV; purulent discharge and Grade V; deep or serious wound infection with or without tissue breakdown (3).

In electrocautery group for Pfannenstiel incision (transversely in the maternal abdomen approximately $2-3 \mathrm{~cm}$ above the symphysis pubis), scalpel used to cut the skin. The subcutaneous tissues and fascia were incised with a high-frequency electric surgical knife. Respectively, fascia transversalis and urachus were dissected approximately $2 \mathrm{~cm}$ by using electrocautery. The rectus muscles were separated using blunt dis- section by fingers together with gentle handling and respect of tissues, to visualize the parietal peritoneum and then entered by bluntly or sharply according to the preference of the surgeon. Then, a standard low-segment transverse uterine incision that $2-3 \mathrm{~cm}$ above the upper edge of the uterovesical fold of peritoneum, performed for the delivery. The expulsion of the fetus and the placenta had occurred respectively before the suturing of the uterine incision by single-layer repair. The fascia and the skin edges were closed with absorbable sutures.

In the control group classically, the same incision technic was performed above the pubic symphysis by a scalpel. After the entrance of the skin, the incision was carried through the lower layers to the fascia using a scalpel for sharp dissection. The fascial incision should be extended laterally using a scalpel or heavy curved Mayo scissors. After the fascia was incised, it should be dissected from the aponeuroses of the rectus abdominis muscles by blunt and sharp dissection in both cephalic and caudal directions. Then the peritoneum entry should be performed. The rest of the operation had been performed in the same way as is described in the first group.

After the procedure had been performed, patients were evaluated on the day of discharge (postpartum day 2), at days 7 to 10 , and at days 30 to 40 . Primarily, the time of the postoperative gas output and neonatal Apgar scores recorded. At each evaluation, the surgical scar was examined for signs of infection, hematoma, seroma, or dehiscence. Patients were asked for the elevation of body temperature at each visit. The patient was discontinued from the study if she was found to have an infection at any site other than the CS scar.

Data analysis was performed using the Statistical Package for Social Sciences version 15 (SPSS Inc, Chicago, IL). The chi-square test was used for categorical variables and the Mann-Whitney U test was used for interval variables. The differences regarded statistically significant in the two-tailed $\mathrm{p}$ value was $<0.05$.

\section{Results}

In this study, there were 37 patients in the electrocautery group and 37 patients in the control group, too. Six patients were excluded in the electrocautery group for not to come controls. Demographic characteristics were found similar between the two groups. There were no statistically significant differences between groups such as age, prenatal care, and parity. The mean age of the patient was $32 \pm 4.8$ at the electrocautery group and $30.8 \pm 5.8$ at the control group (Table I). All patients underwent spinal anesthesia. There were no intraoperative complications in both groups. Surgical procedures' time was similar in both groups, too $(p=0.32)$.

The mean time of postoperative gas output was 33 hours at the cautery group and 21 hours at the control group. Postoperative gas output was significantly earlier at the control group $(p=0.001)$. 
Table I: Comparison of variables according to the groups

\begin{tabular}{llll}
\hline Group & Electrocautery $(n=31)$ & Scalpel $(n=37)$ & $p$ \\
\hline Age & $32 \pm 4.8$ & $30.8 \pm 5.8$ & $0.38^{*}$ \\
Postoperative gas output (hour) & 33 & 21 & $\mathbf{0 . 0 0 1 ^ { * * }}$ \\
Postoperative fever elevation (person) & 1 & 2 & $0.66^{* *}$ \\
Scar complications & $3(9.6 \%)$ & $6(16 \%)$ & $0.69^{*}$ \\
Seroma & 1 & 1 & 1 \\
Hematoma & 1 & 4 & $0.77^{* *}$ \\
Infection & 1 & $3219 \pm 493$ & $0.35^{*}$ \\
The mean birth weight (gram) & $3193 \pm 520$ & 1 & $0.325^{* *}$ \\
Hospitalization neonatal care unit $(\mathrm{n})$ & 0 & $60.65 \pm 5.06$ & \\
Operation time (minute) & $60.16 \pm 10.07$ & & \\
\hline
\end{tabular}

*: Pearson's Chi-Squared test, **: Mann Whitney U test

There were 3 patients of postoperative fever elevation was determined. One of them was in the cautery group and the other two patients were in the control group. There were no statistically significant differences between the groups in terms of postoperative fever $(p=0.66)$.

Ratio of surgical scar complications was $13 \%(n=9)$. The ratio was $9.6 \%(n=3)$ in the cautery group and $16 \%(n=6)$ in the control group. And so there were no statistically significant differences between groups $(p=0.69)$.

The same neonatal findings were found in both groups. The mean birth weight was 3190 gr. at the cautery group and 3219 gr. at the control group. There were no significant differences between newborn Apgar scores ( $1^{\text {st }}$ and $5^{\text {th }}$ minutes $)$. Hospitalization status of infants in the neonatal intensive care unit was evaluated. One newborn was admitted to intensive unit care in the control group. In contrast to this, any newborn was admitted to the intensive care unit in cautery group. Hyperbilirubinemia was the indication of the newborn's hospitalization. Between the groups, there were no statistically significant differences about the admission to neonatal intensive care unit $(p=0.35)$.

\section{Discussion}

Cesarean section is the most frequent surgical procedure in obstetrics (6). Our study is the second in the literature that compares diathermy and scalpel in Pfannenstiel incisions of CS cases but the first one in comparing the primer CS cases. We investigated the incision time, postoperative wound infections and newborn effect in two groups of patients.

Prophylactic usage of antibiotics might theoretically decrease the wound infection rate but not bacterial colonization. So many authors assessed electrocautery over to the scalpel in incisions where skin to the peritoneum operation time in the midline and also one study investigates in transverse surgeries by means of laparotomy (7). All these reports demonstrated the eligibility of electrocautery versus scalpel in operation time and amount of hemorrhage.
Similar to our results but contrary to most of the studies in the literature, Telfer and coworkers compared two groups included a total of 101 patients operated with diathermy and scalpel; authors founded no important significance between electrocautery and scalpel according to the incision time. On the contrary, they showed the presence of a statistical significance in blood loss during incision. It was less in the electrocautery group (8).

In the current study, no significant difference was found in newborn Apgar scores and the need for hospitalization in the neonatal intensive care unit between the two groups. Therefore, the other objective of our study was to find an answer about whether there is a correlation between neonatal complications and incision method? It seems that it is related to the similarity of the operation times. To our knowledge, there is no study that investigates this in the literature. There is a need for future studies that should include neonatal findings.

Cruse's study was an example of prior studies in the literature. They reported that there was a significant relation between wound infections and the usage of electrocautery because of large necrotic-burned tissue left in the wound (9). Cruse and Foord later reported that with reduced destructive tissue, the infection rate in women was similar whether or not diathermy was used (10).

As most of the studies, clinically no statistical importance was determined in wound infections between the two methods like this study. In contrast to this, Amin et al. indicated that the wound healing complications were more related to electrocautery incisions and this would be a reason for varied surgical types (11). Similar to this, Soballe et al. concluded the relation of diathermic incisions with the high incidence of wound infections, indurated margins, and durability of the incision localization in comparison with the scalpel (12).

The relation between postoperative wound complications with the methods of diathermy or scalpel being investigated up to date thus conceived analogous results of wound infections that similar to our study (13-17). 
Ismail et al. have published a systematic review in 2017 December thus included 36 randomized trials, four observational studies, and a quasi-random study, and totally 6422 participants about scalpel and electrocautery comparison. Significantly less blood loss, shorter incisional and operative times were seen in diathermy group. According to this metaanalysis, no statistical differences were detected in terms of overall subjective scar scores or infection rate of the scar. The conclusion of this important meta-analysis pointed out that contemporary data promote the safe usage of diathermy in laparotomic operations (18).

Briefly, no significant difference was detected in this study, between the two methods in terms of neonatal outcomes, wound infections, and incision times. According to these findings, it is suggested that the usage of diathermy instead of a scalpel in surgical incisions because of its easy use without concern of wound complications or high infection rates in comparison with the old belief about it.

\section{Acknowledgments: None}

Funding: No funding to declare.

Declaration of Interest: The authors declare no conflicts of interests.

Authors contribution: EIB: Design, Literature search, Writing manuscript. IG: Design, Supervision, Data collection and/or processing. FCA: Data collection and/or processing, Analysis and/or interpretatiton. MAO: Supervision

\section{References}

1. Mathai M, Hofmeyr GJ. Abdominal surgical incisions for caesarean section. Cochrane Database Syst Rev. 2007; (1):CD004453. doi: 10.1002/14651858.CD004453.

2. Ly J, Mittal A, Windsor J. Systemic review and meta-analysis of cutting diathermy versus scalpel for skin incision. Br J Surg. 2012;99(5):613-20. doi: 10.1002/bjs.8708.

3. Kadyan B, Chavan S, Mann M, Punia P, Tekade S. A prospective study comparing diathermy and steel scalpel in abdominal incisions. Med J DY Patil Univ. 2014; 7:558-63. doi: 10.4103/0975-2870.140382

4. Johnson CD, Serpell JW. Wound infection after abdominal incision with scalpel or diathermy. Br J Surg. 1990;77(6):626-7. doi: 10.1002/bjs.1800770610.

5. Dixon AR, Watkin DF. Electrosurgical skin incision versus conventional scalpel: a prospective trial. J R Coll Surg Edinb. 1990;35(5):299-301. PMID: 2283608

6. Bamigboye AA, Hofmeyr GJ. Closure versus non-closure of the peritoneum at caesarean section. Cochrane Database Syst Rev. 2003;(4): CD000163. doi: 10.1002/ 14651858.

7. Kearns SR, Connolly EM, McNally S, McNamara DA,
Deasy J. Randomized clinical trial of diathermy versus scalpel incision in elective midline laparotomy. Br J Surg. 2001;88(1):41-4. doi: 10.1046/j.1365-2168.2001.018827.x.

8. Telfer JRC, Canning G, Galloway DJ. Comparative study of abdominal incision techniques. Br J Surg. 1993;80(2): 233-5. doi: 10.1002/bjs.1800800238.

9. Cruse PJE. Some factors determining wound infection. A prospective study of 30000 wounds. In: Polk HC Jr, Stone $\mathrm{HH}$, editors. Hospital-Acquired Infections in Surgery. Baltimore: University Park Press;1977. p. 79-85. PMID: 4892316

10. Cruse PJE, Foord R. The epidemiology of wound infection. A 10-year prospective study of 62,939 wounds. Surg Clin North Am. 1980;60(1):27-40. doi: 10.1016/s00396109(16)42031-1.

11. Mir MA, Khan MN, Aziz I, Ismail A. Randomized comparative study of electrosurgical and conventional scalpel incisions in general surgery. Surgery. 2010;20(25):15-22.

12. Soballe PW, Nimbkar NV, Hayward I, Nielsen TB, Drucker WR. Electric cautery lowers the contamination threshold for infection of laparotomies. Am J Surg. 1998; 175(4):263-6. doi: 10.1016/s0002-9610(98)00020-8.

13. Ahmed NZ, Ahmed A. Meta-analysis of the effectiveness of surgical scalpel or diathermy in making abdominal skin incisions. Ann Surg. 2011;253(1):8-13. doi: 10.1097/ SLA.0b013e3181ff461f.

14. Shivagouda P, Gogeri BV, Godhi AS, Metgud SC. Prospective randomized control trial comparing the efficacy of diathermy incision versus scalpel incision over skin in patients undergoing inguinal hernia repair. Recent Res Sci Tech. 2010;2(8):44-7.

15. Hemsell DL, Hemsell PG, Nobles B, Johnson ER, Little BB, Heard M. Abdominal wound problems after hysterectomy with electrocautery vs. scalpel subcutaneous incision. Infect Dis Obstet Gynecol. 1993;1(1):27-31. Doi: 10.1155/S1064744993000079.

16. Upadhyay S, Bansal N. Electrocautery versus scalpel incision in inguinal hernioplasty. RJPBCS. 2013;4(4):499503.

17. Elbohoty AE, Gomaa MF, Abdelaleim M, Abd-El-Gawad M, Elmarakby M. Diathermy versus scalpel in transverse abdominal incision in women undergoing repeated cesarean section: a randomized controlled trial. J Obstet Gynaecol Res. 2015;41(10):1541-6. doi: 10.1111/jog.127 76.

18. Ismail A, Abushouk AI, Elmaraezy A, Menshawy A, Menshawy E, Ismail M, et al. Cutting electrocautery versus scalpel for surgical incisions: a systematic review and meta-analysis. J Surg Res. 2017;220:147-63. doi: 10.1016 /j.jss.2017.06.093. 